\title{
EXACT SOLUTIONS FOR A BENJAMIN EQUATION WITH HIGHER-ORDER NONLINEARITY AND VARIABLE
}

\author{
Cesar A. Gómez S. \\ ${ }^{1}$ Departamento de Matemáticas \\ Universidad Nacional de Colombia \\ Bogotá, Kra. 30 No. 45-03, of. 209-404, COLOMBIA
}

\begin{abstract}
We use a improved tanh-coth method to obtain exact traveling wave solutions for a Benjamin equation with higher-order nonlinearity and variable coefficients. The obtained solutions are given in more general form than those derived by other authors using other computational methods. The advantage of the calculus made in this paper is that we can consider the coefficients depending on the time variable, which give us many other models from which the generalized Benjamin equation is a particular case. On the other hand, the solutions obtained here can be used to solve other important models of nonlinear equations with variable coefficients, only making a brief change. We explain this fact with one example.
\end{abstract}

AMS Subject Classification: 35C05

Key Words: Improved tanh-coth method; Higher-order nonlinearity; Generalized Benjamin equation; KdV equation; modified KdV equation; periodic and soliton solutions.

\section{Introduction}

The study of nonlinear partial differential equations (NLPDE's) with higherorder nonlinearity, is a task not easy to make. However, the use of modern symbolic and computational packages as Mathematica or Maple, give us the possibility of obtain exact traveling wave solutions for some few of this models. Many computational methods have been developed in the last decades with the

$\begin{array}{lrc}\text { Received: } & \text { March 16, } 2016 & \text { (C) } 2016 \text { Academic Publications, Ltd. } \\ \text { Revised: } & \text { July 7, } 2016 & \text { url: www.acadpubl.eu } \\ \text { Published: } & \text { October 21, } 2016 & \end{array}$


aim to solve many nonlinear partial differential equations (NLPDE's). One of the methods used in this direction, is given by the improved tanh-coth method [1] which give us solutions in a more general form than the obtained by the extended tanh method [2].

The main objective of this paper is the search of exact solution to the following nonlinear partial differential equation with higher-order nonlinearity

$$
u_{t t}+\alpha(t)\left(u^{n} u_{x}\right)_{x}+\beta(t) u_{x x x x}=0
$$

being $\alpha(t), \beta(t)$ arbitrary functions depending of the time $t, u(x, t)$ the unknown function and $n$ is a positive integer number. The generalized Benjamin equation is obtained in the case $\alpha(t)=\alpha$ and $\beta(t)=\beta$ are arbitrary constants. Solutions for 1 in this last case, have been obtained using the extended tanh method and the $G^{\prime} / G$-method in [3], [4] respectively. We will use the improved tanh-coth method for obtain exact solutions to Eq.(1) and we will observe that with a brief change, the solutions obtained can be used for solve (from the point of view of exact solutions) the following nonlinear partial differential equation with higher-order nonlinearity [5]

$$
u_{t}(x, t)+\alpha(t) u^{n}(x, t) u_{x}(x, t)+\beta(t) u_{x x x}(x, t)=0,
$$

which have two important particular cases, used in nonlinear phenomena: The Korteweg-de Vries equation [6]

$$
u_{t}(x, t)+6 u(x, t) u_{x}(x, t)+u_{x x x}(x, t)=0,
$$

and the modified Korteweg-de Vries equation [7]

$$
u_{t}(x, t)+k_{1} u^{2}(x, t) u_{x}(x, t)+u_{x x x}(x, t)=0 .
$$

Other two models used in dynamic of fluids, (see [8], [9] and references therein), which are particular cases of (2), are the following

$$
\left\{\begin{array}{l}
u_{t}(x, t)+k_{1} t^{n} u^{2}(x, t)+k_{2} t^{m} u_{x x x}(x, t)=0, \\
\text { and } \\
u_{t}(x, t)+k_{1} u^{n}(x, t) u_{x}(x, t)+u_{x x x}(x, t)=0 .
\end{array}\right.
$$




\section{Exact traveling wave solutions to Eq.(1)}

With the aim to illustrate the improved tanh-coth method, we consider that the solution of Eq. (1) have the form

$$
\left\{\begin{array}{l}
u(x, t)=v(\xi) \\
\xi=x+\lambda t+\xi_{0}
\end{array}\right.
$$

where $\lambda$ is the speed of the wave and $\xi_{0}$ an arbitrary constant. Substitution of (6) into (1) and after one integration, we obtain the following ordinary differential equation

$$
\lambda^{2} v^{\prime}(\xi)+\alpha(t) v^{n}(\xi) v^{\prime}(\xi)+\beta(t) v^{\prime \prime \prime}(\xi)=0
$$

where, as we are searching exact solutions, we have taken the integration constant as zero. One more integration give us the equation

$$
\lambda^{2} v(\xi)+\frac{\alpha(t)}{n+1} v(\xi)^{n+1}+\beta(t) v^{\prime \prime}(\xi)=0
$$

where, as previous case, we have taken the integration constant as zero. Now, with the change of variable

$$
v(\xi)=V^{\frac{1}{n}}(\xi)
$$

Eq.(8) reduces to

$$
\lambda^{2} n^{2}(n+1) V^{2}(\xi)+\alpha(t) n^{2} V^{3}(\xi)+\beta(t)\left[\left(1-n^{2}\right) V^{\prime}(x i)^{2}+n(n+1) V(\xi) V^{\prime \prime}(\xi)\right]=0
$$

The improved tanh-coth method use as solution of (10) the expansion

$$
V(\xi)=\sum_{i=0}^{M} a_{i}(t) \phi(\xi)^{i}+\sum_{i=M+1}^{2 M} a_{i}(t) \phi(\xi)^{M-i}
$$

where $M$ is a positive integer determined by balancing and $\phi=\phi(\xi)$ satisfies the Riccati equation

$$
\phi^{\prime}(\xi)=r(t)+q(t) \phi(\xi)+p(t) \phi(\xi)^{2} .
$$

The $a_{i}(t), i=1,2, \ldots, 2 M, r(t), p(t), q(t)$ are functions depending only of the variable $t$ to be determined later and the solution of (12) in the case $q(t)^{2}-$ $4 p(t) q(t) \neq 0$ is given by (see $[10])$ : 


$$
\phi(\xi)=\frac{-\sqrt{q(t)^{2}-4 p(t) r(t)} \tanh \left[\frac{1}{2} \sqrt{q(t)^{2}-4 p(t) q(t)} \xi+\xi_{0}\right]-q(t)}{2 p(t)} .
$$

Substituting (11) into (10), and balancing $V(\xi) V^{\prime \prime}(\xi)$ with $V(\xi)^{3}$ we obtain

$$
2 M+2=3 M,
$$

so that

$$
M=2 .
$$

Therefore, (11) reduces to

$$
V(\xi)=a_{0}(t)+a_{1}(t) \phi(\xi)+a_{2}(t)(\phi(\xi))^{2}+a_{3}(t)(\phi(\xi))^{-1}+a_{4}(t)(\phi(\xi))^{-2} .
$$

Now, substituting (14) into (10), taking in account (12) and equating to zero the coefficients of all powers of $\phi(\xi)$, we get a set of algebraic equations for $a_{0}(t), a_{1}(t), a_{2}(t), a_{3}(t), a_{4}(t), r(t), q(t), p(t)$. Solving the system with aid the Mathematica we obtain a lot of solutions, from which, for the sake of simplicity and with the aim to observe the different types of expressions that we can obtain, we consider only the following three cases:

First Case:

$$
\left\{\begin{array}{l}
r(t)=\frac{q(t)^{2} \beta(t)+\lambda^{2} n^{2}}{4 \beta(t) p(t)}, a_{0}(t)=-\frac{\left(n^{2}+3 n+2\right)\left(q(t)^{2} \beta(t)+\lambda^{2} n^{2}\right)}{2 \alpha(t) n^{2}} \\
a_{1}(t)=-\frac{2 q(t) \beta(t) p(t)\left(n^{2}+3 n+2\right)}{\alpha(t) n^{2}}, a_{2}(t)=-\frac{2 \beta(t) p(t)^{2}\left(n^{2}+3 n+2\right)}{\alpha(t) n^{2}} \\
a_{3}(t)=0, \quad a_{4}(t)=0 .
\end{array}\right.
$$

With respect to this values, and taking into account (13) we have

$$
\phi(\xi)=-\frac{q(t)+\sqrt{-\frac{\lambda^{2} n^{2}}{\beta(t)}} \tanh \left(\frac{1}{2} \sqrt{-\frac{\lambda^{2} n^{2}}{\beta(t)}} \xi\right)}{2 p(t)} .
$$

Finally, by (14), (9) and (6) we have that one solution of (1) is given by

$$
u(x, t)=v(\xi)=\left[a_{0}(t)+a_{1}(t) \phi(\xi)+a_{2}(t) \phi(\xi)^{2}\right]^{\frac{1}{n}},
$$

where $a_{0}(t), a_{1}(t), a_{2}(t)$ are given by $(15), \phi(\xi)$ given by $(16), p(t), q(t)$ arbitrary functions in the variable $t, \xi$ given by (6), where $\xi_{0}$ and $\lambda$ are arbitrary parameters. 
Second case:

$$
\left\{\begin{array}{l}
p(t)=\frac{q(t)^{2} \beta(t)+\lambda^{2} n^{2}}{4 r(t) \beta(t)}, \quad a_{0}(t)=-\frac{(n+1)(n+2)\left(q(t)^{2} \beta(t)+\lambda^{2} n^{2}\right)}{2 \alpha(t) n^{2}} \\
a_{3}(t)=-\frac{2 r(t) q(t) \beta(t)\left(n^{2}+3 n+2\right)}{\alpha(t) n^{2}}, \quad a_{4}(t)=-\frac{2 r(t)^{2} \beta(t)\left(n^{2}+3 n+2\right)}{\alpha(t) n^{2}} \\
a_{3}(t)=a_{4}(t)=0 .
\end{array}\right.
$$

With respect to this values, and taking into account (13) we have

$$
\phi(\xi)=\frac{2 r(t) \beta(t)\left(-q(t)-\sqrt{-\frac{\lambda^{2} n^{2}}{\beta(t)}} \tanh \left(\frac{1}{2} \sqrt{-\frac{\lambda^{2} n^{2}}{\beta(t)}} \xi\right)\right)}{q(t)^{2} \beta(t)+\lambda^{2} n^{2}},
$$

so that, as in the previous case, the solution to (1) is given by,

$$
u(x, t)=\left[a_{0}(t)+a_{3}(t) \phi(\xi)^{-1}+a_{4}(t) \phi(\xi)^{-2}\right]^{\frac{1}{n}}
$$

where $a_{0}(t), a_{3}(t), a_{4}(t)$ are given by $(18), \phi(\xi)$ given by $(19), q(t), r(t)$ arbitrary functions in the variable $t, \xi$ given by (6), with $\xi_{0}$ and $\lambda$ arbitrary parameters.

Third case:

$$
\left\{\begin{array}{l}
r(t)=\frac{\lambda^{2} n^{2}}{16 \beta(t) p(t)}, \quad a_{0}(t)=-\frac{\lambda^{2}\left(n^{2}+3 n+2\right)}{4 \alpha(t)} \\
a_{2}(t)=-\frac{2 \beta(t) p(t)^{2}\left(n^{2}+3 n+2\right)}{\alpha(t) n^{2}}, \quad a_{4}(t)=-\frac{\lambda^{4} n^{2}\left(n^{2}+3 n+2\right)}{128 \alpha(t) \beta(t) p(t)^{2}} \\
a_{1}(t)=a_{3}(t)=\beta(t)=0 .
\end{array}\right.
$$

With respect to this values, and taking into account (13) we have

$$
\phi(\xi)=-\frac{\sqrt{-\frac{\lambda^{2} n^{2}}{\beta(t)}} \tanh \left(\frac{1}{4} \sqrt{-\frac{\lambda^{2} n^{2}}{\beta(t)}} \xi\right)}{4 p(t)}
$$

so that, as in the previous case, the solution to (1) is given by,

$$
u(x, t)=\left[a_{0}(t)+a_{2}(t) \phi(\xi)^{2}+a_{4}(t) \phi(\xi)^{-2}\right]^{\frac{1}{n}}
$$

where $a_{0}(t), a_{2}(t), a_{4}(t)$ are given by $(21), \phi(\xi)$ given by $(22), p(t)$ arbitrary function in the variable $t, \xi$ given by (6), with $\xi_{0}$ and $\lambda$ arbitrary parameters. It is clear that varying the parameter $\xi_{0}$ we can obtain many periodic and soliton solutions.

Finally, note that if we take $\lambda^{2}$ instead of $\lambda$ in (6), the substitution of resulting expression into (2) give us (7). Therefore traveling wave solutions to 
(7) can be derived from the solutions obtained to (1) using $\xi=x+\lambda^{2} t+\xi_{0}$. The following example illustrate this fact:

The following is a solution of the algebraic system mentioned previously

$$
\left\{\begin{array}{l}
a_{0}(t)=-\frac{\lambda^{2}\left(n^{2}+3 n+2\right)}{2 \alpha(t)}, \quad a_{4}(t)=-\frac{2 r(t)^{2} \beta(t)\left(n^{2}+3 n+2\right)}{\alpha(t) n^{2}} \\
p(t)=\frac{\lambda^{2} n^{2}}{4 r(t) \beta(t)}, \quad a_{1}(t)=a_{2}(t)=a_{3}(t)=q(t)=0
\end{array}\right.
$$

The corresponding solution to (12) is given by

$$
\phi(\xi)=-\frac{r(t) \beta(t) \sqrt{-\frac{\lambda^{2}}{\beta(t)}} \tanh \left(\sqrt{-\frac{\lambda^{2}}{\beta(t)}} \xi\right)}{\lambda^{2}} .
$$

Then, according with the method, the respective solution to (1) have the form

$$
u(x, t)=\left[-\frac{\lambda^{2}\left(n^{2}+3 n+2\right)}{2 \alpha(t)}-\frac{2 r(t)^{2} \beta(t)\left(n^{2}+3 n+2\right)}{\alpha(t) n^{2}} \phi(x+\lambda t)^{-2}\right]^{\frac{1}{n}},
$$

with $\phi(\xi)$ given by (25). In this order of ideas, one solution to (6) can be expressed as

$$
u(x, t)=\left[-\frac{\lambda^{2}\left(n^{2}+3 n+2\right)}{2 \alpha(t)}-\frac{2 r(t)^{2} \beta(t)\left(n^{2}+3 n+2\right)}{\alpha(t) n^{2}} \phi\left(x+\lambda^{2} t\right)^{-2}\right]^{\frac{1}{n}}
$$

So that, solutions to (3), (4) and (5) are obtained from this last, taking the respective values of the parameters.

\section{Conclusions}

Using the improved tanh-coth method, we have obtained solutions to a generalized Benjamin equation with variable coefficients. The solutions are in a more general form that those obtained in [3], [4]. The variation of the parameter $\xi_{0}$ give us the possibility of obtain many other form of the solutions such as soliton or periodic solution type. The advantage of the calculus here presented is that we can to change $\lambda$ by $\lambda^{2}$ in all solutions obtained for (1) to obtain exact solutions for a variety of important equations such as the KdV and the modified $\mathrm{KdV}$ equations as well as other type of nonlinear equations with variable coefficients, used in dynamic of fluids. 


\section{References}

[1] C. A. Gomez S., Closed form solutions for a generalized Benjamin-Bona-Mahony-Burgers equation with higher-order nonlinearity, Appl. Math, and Comp., 234 (2014), 618-622, doi: 10.1016/j.amc.2014.02.064.

[2] A.M. Wazwaz, The extended tanh method for new soliton solutions for many form of the fifth-order KdV equations, Appl. Math and Comp,, 84, No. 2 (2007), 1002-1014. (http://DOI: 10.1016/j.amc.2006.07.002.

[3] N.Taghizadeh, M. Mirzazadeh and S.R.Moosavi Noori, Exact solutions of the Generalized Benajmin Equations and (3+1)-Dimensional Gkp Equation by the Extended Tanh Method, Applications and Applied Mathematics: An international Journal (AAM), 7, No. 1 (2012), 175-187.

[4] Hua Gao, Genhu Di, Exact solutions to the generalized Benjamin Equation, J. of Applied Mathematis and physics, 2 (2014), 671-676, doi: 10.4236/jamp.2014.27074.

[5] C. A. Gomez S., On a KdV equation with higher-order nonlinearity: Traveling wave solutions , Journal of Computational and Applied Mathematics, 235 (2011), 5330-5332, doi: 10.1016/j.cam.2011.05.028.

[6] G.B. Whitham, Linear and Nonlinear waves, Wiley, New York, 1974.

[7] R.M. Miura, The Korteweg-de Vries equations and generalizations. A remarkable explicit nonlinear transformation, J. Math. Phys., 9 (1968), 1202-1204, doi: 10.1063/1.1664700)

[8] N. Nirmala, M.J. Vedan and B.V. Baby, Auto-Bäcklund transformation, Lax Pairs Painlevé property or a variable coefficient Korteweg-de Vries equation, J. Math. Phys., 27 (1986), 2640-2646, doi: 10.1063/1.527282)

[9] Liu C. Yang, The applications of bifurvation method to a higher-order KdV equation, Math. Anal. Appl., 275 (2012), 1-12, doi: 10.1016/S0022-247X(02)00210-X)

[10] C. A. Gomez S and A. Salas, Special symmetries to standard Riccati equations and applications, Appl. Math, and Comp., 216, No. 10 (2010), 3089-3096, doi: 10.1016/j.amc.2010.04.039) 
